\title{
ELECTROMAGNETIC SCATTERING ON NONSMOOTH DOMAINS
}

\author{
Marius Mitrea
}

\begin{abstract}
We initiate the study of the boundary value problems for Maxwell equations in arbitrary Lipschitz domains by means of layer potential techniques.
\end{abstract}

\section{Introduction}

The central problem in direct (classical) electromagnetic scattering theory, i.e. describing the wave propagation of the the electric and magnetic fields $(E, H)$, is of fundamental importance both from a theoretical and a practical point of view. Over the years the subject has continuously received a great deal of attention; detailed accounts on these matters can be found in [6],[4].

The boundary value problems for the time-independent form of Maxwell equations in smooth domains have been solved for the first time in the early 1950's [2],[13],[15] in terms of singular layer potential integral operators. Due to the smoothness of the boundary of the domain, these integrals are actually only weakly singular, hence giving rise to compact operators for which the Fredholm theory is readily applicable. More recently, these techniques have been refined to treat $C^{1}$ domains, too ([12]).

This note reports on progress made in the direction of employing the layer potential techniques for solving boundary value problems related to the Maxwell system on Lipschitz domains. In the time-independent case we shall treat the Dirichlet problem on arbitrary Lipschitz domains in three space dimensions and even in the higher dimensional setting, as well as the more general case of first order elliptic complexes satisfying certain symmetry conditions. The parabolic form of the Maxwell equation on Lipschitz cylinders is also considered. An immediate difficulty is that compactness arguments are no longer directly applicable on Lipschitz boundaries.

Received June 7, 1994

1991 Mathematics Subject Classification. Primary 42B20, 45F15; Secondary 35J55, $35 \mathrm{~K} 20$.

Key words and phrases. Scattering theory, Maxwell equations, Lipschitz domains. Supported in part by NSF. 
This effort builds on the fundamental work of Dahlberg, Fabes, Jerison, Kenig, Verchota for the Laplacian and its further refinements to the system of elastostatics and to the linearized Stokes system. One of the key ingredients of this approach is establishing the relevant Rellich identities for the Maxwell system.

A distinct characteristic of the problems at hand is that the boundary data (necessarily) exhibit regularity properties of a fairly intricate geometric nature (as opposed to other cases mentioned above). In particular, arguments involving flattening the boundary of the domain, as in [14] or [5], are not suited for the problems under discussion. We overcome this difficulty by making a careful analysis of the spectrum of the Maxwell boundary integral operator. Among other things, we are able to relate its spectral radius to that of the classical double layer harmonic potential operator.

More applications and details of the results announced here will appear elsewhere. Part of them have been obtained in collaboration with other people and will be published as joint work.

\section{Statement of main results.}

For the sake of brevity we shall not attempt to give complete definitions unless absolutely necessary. For more information we refer the reader to e.g. [9],[10] whose notation and terminology we shall employ in the sequel.

Let $\Omega$ be a bounded Lipschitz domain in $R^{3}$. The boundary value problem for the Maxwell system, in the strong sense of Calderón [2], resides in finding a pair $(E, H)$ of vector fields which are smooth inside $\Omega$ and satisfy

$$
(\mathcal{M})\left\{\begin{array}{l}
\text { curl } E-i k H=0 \text { on } \Omega, \\
\text { curl } H+i k E=0 \text { on } \Omega, \\
E^{*}, H^{*} \in L^{2}(\partial \Omega) \\
n \times\left. E\right|_{\partial \Omega}=A
\end{array}\right.
$$

Here $k \in \mathrm{C}$ depends solely on the electric and magnetic characteristics of the medium in $\mathrm{R}^{3}$. For simplicity, throughout the paper we shall assume that $\operatorname{Im} k>|R e k|$. Also, $n$ is the unit normal defined a.e. on $\partial \Omega, *$ is the non-tangential maximal operator and the boundary trace is taken in the non-tangential limit sense (see [9],[10]). that

Let $L_{\text {tan }}^{2, D i v}(\partial \Omega)$ be the space of all tangential vector fields on $\partial \Omega$ such

$$
\|A\|_{L_{t a n}^{2, D i v}(\partial \Omega)}:=\sup \left\{\left|\int_{\partial \Omega}\right| A|\varphi d \sigma|+\left|\int_{\partial \Omega}\left\langle\nabla_{\tan } \varphi, A\right\rangle d \sigma\right|\right\}<+\infty
$$


where the supremum is taken over all scalar-valued functions $\varphi \in C^{1}\left(\mathrm{R}^{3}\right)$ with $\|\varphi\|_{L^{2}(\partial \Omega)}=1$, and $\nabla_{\text {tan }}$ is the usual tangential gradient on $\partial \Omega$.

Theorem 1. For any $A \in L_{\text {tan }}^{2, D i v}(\partial \Omega)$ the problem $(\mathcal{M})$ has a unique solution. This solution also satisfies

$$
\left\|E^{*}\right\|_{L^{2}(\partial \Omega)}+\left\|H^{*}\right\|_{L^{2}(\partial \Omega)} \leq C\|A\|_{L_{\text {tan }}^{2, D i v}(\partial \Omega)},
$$

and

$$
\|E\|_{W^{1 / 2}(\Omega)}+\|H\|_{W^{1 / 2}(\Omega)} \approx\|A\|_{L_{t a n}^{2, D i v}(\partial \Omega)} .
$$

For $(\mathcal{M})$ to be solvable, $A \in L_{\text {tan }}^{2, D i v}(\partial \Omega)$ is a necessary condition, too. One can actually solve a somewhat more general problem which we now describe.

Theorem 2. The following boundary value problem has a unique solution:

$$
\left\{\begin{array}{l}
\left(\triangle+k^{2}\right) E=0 \text { on } \Omega \\
\|\mid E\|:=\left\|E^{*}\right\|_{L^{2}(\partial \Omega)}+\left\|(\operatorname{curl} E)^{*}\right\|_{L^{2}(\partial \Omega)}+\left\|(\operatorname{div} E)^{*}\right\|_{L^{2}(\partial \Omega)}<+\infty \\
n \times\left. E\right|_{\partial \Omega}=A \in L_{\text {tan }}^{2, D i v}(\partial \Omega) \\
\left.(\operatorname{div} E)\right|_{\partial \Omega}=f \in L^{2}(\partial \Omega)
\end{array}\right.
$$

This solution also satisfies $\left\||E \|| \leq C\left(\|A\|_{L_{\text {tan }}^{2, D i v}(\partial \Omega)}+\|f\|_{L^{2}(\partial \Omega)}\right)\right.$.

In addition, $(\nabla(\operatorname{div} E))^{*} \in L^{2}(\partial \Omega)$ if and only if $f \in L^{2,1}(\partial \Omega)$. In this latter case, $\left\|(\nabla(\operatorname{div} E))^{*}\right\|_{L^{2}} \leq C\left(\|f\|_{L^{2}}+\left\|\nabla_{\text {tan }} f\right\|_{L^{2}}\right)$.

The problem $(\mathcal{M})$ generalizes to higher dimensions as follows. Given a Lipschitz domain $\Omega$ in $\mathrm{R}^{m}$ and $0 \leq l \leq m-1$, determine a $(l+1)$-form $E$ and a $l$-form $H$ having smooth coefficients in $\Omega$ and such that

$$
\left(\mathcal{M}_{m}\right)\left\{\begin{array}{l}
\delta E-i k H=0 \text { on } \Omega \\
d H+i k E=0 \text { on } \Omega \\
E^{*}, H^{*} \in L^{2}(\partial \Omega) \\
\left.n \vee E\right|_{\partial \Omega}=A
\end{array}\right.
$$

Here $d$ is the usual exterior derivative operator, $\delta$ stands for its formal transpose, $n$, the outward unit normal, is canonically identified with a 1form, and $\vee$ is the interior product of forms. For $\left(\mathcal{M}_{m}\right)$ to be solvable, the $l$-form $A$ must be tangential, i.e. $n \vee A=0$ a.e. on $\partial \Omega$, have square integrable coefficients on $\partial \Omega$, and satisfy

$$
\||A|\|_{2}:=\sup \left|\int_{\partial \Omega}\langle d \psi, A\rangle d \sigma\right|<+\infty
$$

where the supremum is taken over the set of all $(l-1)$-forms $\psi$ with coefficients in $C^{1}\left(\mathrm{R}^{m}\right)$ normalized so that $\|\psi\|_{L^{2}(\partial \Omega)}=1$. 
Theorem 3. For any $A$ as above, the problem $\left(\mathcal{M}_{m}\right)$ has a unique solution. Moreover, this solution satisfies

$$
\left\|E^{*}\right\|_{L^{2}(\partial \Omega)}+\left\|H^{*}\right\|_{L^{2}(\partial \Omega)} \leq C\left(\|A\|_{L^{2}(\partial \Omega)}+\||A|\|_{2}\right) .
$$

Another problem of interest is that of determining a harmonic field in a Lipschitz domain having a prescribed tangential component on the boundary. In concrete terms, one looks for a $(l+1)$-form $E$ so that

$$
\left(\mathcal{D}_{m}\right)\left\{\begin{array}{l}
d E=0=\delta E \text { on } \Omega, \\
E^{*} \in L^{2}(\partial \Omega) \\
\left.n \vee E\right|_{\partial \Omega}=A
\end{array}\right.
$$

Theorem 4. Assume that $\Omega$ is a special Lipschitz domain in $R^{m}$. Then $\left(\mathcal{D}_{m}\right)$ is uniquely solvable if and only if $A$ is a tangential l-form with square integrable coefficients on $\partial \Omega$ and such that

$$
\int_{\partial \Omega}\langle d \psi, A\rangle d \sigma=0
$$

for any $(l-1)$-forms $\psi$ with coefficients in $C_{0}^{1}\left(R^{m}\right)$.

A similar result, this time prescribing the normal component of $E$ on the boundary, is also valid. In fact, these two boundary value problems are dual to each other via the Hodge $*$-isomorphism. It is interesting to note that these results naturally extend those of Dahlberg, Jerison, Kenig and Verchota concerning the $L^{2}$-Dirichlet and Neumann problem for harmonic functions. Moreover, results of this type can also be obtained for harmonic tensors, i.e. differential forms such that $d \delta E=0=d E$ or $\delta d E=0=\delta E$.

We now describe the parabolic version of the Maxwell equations in Lipschitz cylinders. Let $D_{t}^{1 / 2}, I_{1 / 2}$ stand, respectively, for the half-order fractional derivative and fractional integration operators in the time variable. The boundary value problem for the diffusion equation of the electromagnetic fields $(\mathcal{E}, \mathcal{H})=\left(\left(\mathcal{E}_{i}(x, t)\right)_{i=1}^{3},\left(\mathcal{H}_{i}(x, t)\right)_{i=1}^{3}\right)$ in the domain $\Omega \times \mathrm{R}$ in $\mathrm{R}^{4}$ is (cf. [6])

$$
\left(\mathcal{M}_{\text {par }}\right)\left\{\begin{array}{l}
\mathcal{E}, \mathcal{H} \in C^{\infty}(\Omega \times(0, \infty)) \\
\left.\mathcal{E}\right|_{t=0}=\left.\mathcal{H}\right|_{t=0}=0 \text { on } \Omega \\
\mathcal{E}^{*},\left(D_{t}^{1 / 2} \mathcal{H}\right)^{*} \in L^{2}(\partial \Omega \times(0, \infty)), \\
\text { curl } \mathcal{E}-\partial_{t} \mathcal{H}=0 \text { on } \Omega \times(0, \infty), \\
\text { curl } \mathcal{H}+\mathcal{E}=0 \text { on } \Omega \times(0, \infty), \\
n \times\left.\mathcal{E}\right|_{\partial \Omega \times(0, \infty)}=\mathcal{A}
\end{array}\right.
$$


Theorem 5. A necessary and sufficient condition for the problem $\left(\mathcal{M}_{\text {par }}\right)$ to be uniquely solvable is that $\mathcal{A}$ is a tangential, square integrable vector field on $\partial \Omega \times(0, \infty)$ for which

$$
\sup \left|\int_{0}^{\infty} \int_{\partial \Omega}\left\langle\nabla_{\tan } \psi, I_{1 / 2} \mathcal{A}\right\rangle d \sigma\right|<+\infty,
$$

with supremum taken over all functions $\psi \in C_{0}^{1}\left(R^{4}\right)$ with

$$
\|\psi\|_{L^{2}(\partial \Omega \times R)}=1 .
$$

\section{Sketch of proofs and further results}

- The problem $(\mathcal{M})$ essentially reduces to proving that $\pm \frac{1}{2}+M$ are isomorphisms of $L_{\text {tan }}^{2, D i v}(\partial \Omega)$, where the principal value singular integral operator $M$ is given by $M A:=n \times \operatorname{curl} \mathcal{S} A$ with $\mathcal{S}$ standing for the simple-layer acoustic-potential operator on $\partial \Omega$. Since, clearly, $\lambda+M$ has index zero for $\lambda$ sufficiently large, the main idea of proof is to show that $\lambda+M$ is a semi-Fredholm operator on $L_{\text {tan }}^{2, D i v}(\partial \Omega)$ for each $\lambda \in \mathrm{R} \backslash\left(-\frac{1}{2}, \frac{1}{2}\right)$.

Let us briefly indicate how this can be done for the critical values $\lambda=$ $\pm \frac{1}{2}$. The key step is establishing the estimate

$$
\|(-1 / 2+M) A\|_{L_{t a n}^{2, D i v}(\partial \Omega)} \approx\|(1 / 2+M) A\|_{L_{t a n}^{2, D i v}(\partial \Omega)} .
$$

In doing so, the main ingredient is a suitable Rellich type identity. More specifically, for any smooth vector fields $E, \Theta$, we have

$$
\begin{gathered}
\operatorname{Re}\left(\int_{\partial \Omega} \frac{1}{2}|E|^{2}\langle\Theta, n\rangle d \sigma-\int_{\partial \Omega}\langle\bar{E}, \Theta\rangle\langle E, n\rangle d \sigma\right) \\
=\operatorname{Re}\left(\iint_{\Omega} \frac{1}{2}|E|^{2} \operatorname{div} \Theta-\langle\bar{E}, \Theta\rangle \operatorname{div} E-\langle\bar{E},(\nabla \Theta) E\rangle+\langle\operatorname{curl} E, \bar{E} \times \Theta\rangle\right) .
\end{gathered}
$$

Here $(\nabla \Theta) E$ is the matrix $\left\{\partial_{i} \Theta_{j}\right\}_{i, j}$ acting on $E$. For $(E, H)$ as in $(\mathcal{M})$, this amounts to

$$
\|n \times E\|_{L_{t a n}^{2, D i v}(\partial \Omega)} \approx\|n \times H\|_{L_{t a n}^{2, D i v}(\partial \Omega)} .
$$

Expressing $E, H$ as appropriate layer potentials, (1) follows. Note that (3) is the Lipschitz domain version of the fact that the so called voltageto-current map $\Lambda: n \times E \mapsto n \times H$ is an isomorphism.

Let us point out that our approach also gives that the spectral radius of $M$ on $L_{\text {tan }}^{2, D i v}(\partial \Omega)$ is $<\frac{1}{2}$ provided the spectral radius of the classical double layer harmonic potential operator acting on the space of square integrable functions with zero integral mean is so. While still open for 
general Lipschitz domains, this latter fact is known to be true for bounded convex domains or Lipschitz domains with small Lipschitz constant ([7]). Therefore, whenever this holds true, we have

$$
\left( \pm \frac{1}{2}+M\right)^{-1}= \pm 2 \sum_{j=0}^{\infty}(\mp 2 M)^{j}
$$

with convergence in the strong operator norm.

As in the case of the heat equation ([1]), taking a partial Fourier transform in the time variable essentially reduces $\left(\mathcal{M}_{\text {par }}\right)$ to the study of a family of elliptic boundary value problems with one parameter. Then the main difficulty lies in obtaining estimates with adequate control on the parameter. Theorem 5 is new even on cylinders with smooth profile. Certainly, it would be of interest to analyze the corresponding problem in time-varying domains.

The higher dimensional analogue of $(2)$ is

$$
\begin{gathered}
R e \iint_{\Omega} \frac{1}{2}|E|^{2} \operatorname{div} \Theta+\langle\delta E, \Theta \vee \bar{E}\rangle+\langle d E, \Theta \wedge \bar{E}\rangle-\sum_{j}\left\langle d \Theta_{j} \vee E, e_{j} \vee \bar{E}\right\rangle \\
=\int_{\partial \Omega} \frac{1}{2}|E|^{2}\langle\Theta, n\rangle-\operatorname{Re} \int_{\partial \Omega}\langle\Theta \vee \bar{E}, n \vee E\rangle .
\end{gathered}
$$

Here $\left\{e_{j}\right\}_{j=1}^{m}$ is the standard basis in $\mathrm{R}^{m}, E$ is a differential form on $\Omega$ and $\Theta=\left\{\Theta_{j}\right\}_{j=1}^{m}$ vector field in $\mathrm{R}^{m}$, with smooth, real-valued components. Note that, in the particular case when $E=d u$, with $u$ a harmonic function, this identity reduces to the one used by Jerison, Kenig and Verchota. Furthermore, it also has important connections with the Clifford algebra formalism (cf. [11]) and problems arising in spectral geometry.

As for Theorem 4, let us remark that the above identity also implies

$$
\begin{aligned}
\|E\|_{L^{2}(\partial \Omega) \leq} \leq & C \min \left\{\|n \wedge E\|_{L^{2}(\partial \Omega)},\|n \vee E\|_{L^{2}(\partial \Omega)}\right\} \\
& +C\left|\int_{\partial \Omega}\langle\delta E, n \vee \bar{E}\rangle\right|+C\left|\int_{\partial \Omega}\langle d E, n \wedge \bar{E}\rangle\right| .
\end{aligned}
$$

Now, if $E$ is a harmonic field (i.e. $d E=0=\delta E$ ), then (4) reduces to $\left\|E_{\text {tan }}\right\|_{L^{2}(\partial \Omega)} \approx\left\|E_{\text {nor }}\right\|_{L^{2}(\partial \Omega)}$ which, for the harmonic case, plays a role analogous to that of $(3)$.

Finally, let us note that our main identities continue to hold on manifolds, too. In fact, they also carry over to the more general setting of first-order, linear elliptic complexes satisfying certain symmetry conditions. More concretely, if $\left(\sigma\left(d_{j} ; \cdot\right)\right)_{j}$ is the sequence of associated symbols 
of a first order elliptic complex $\left(d_{j}\right)_{j}$, then the required assumption is that, for each $j$, the bilinear form

$$
\Pi_{j}(\xi, \mu):=\sigma\left(d_{j-1} ; \xi\right) \sigma\left(d_{j-1}^{t} ; \mu\right)+\sigma\left(d_{j}^{t} ; \mu\right) \sigma\left(d_{j} ; \xi\right)
$$

is symmetric in $\xi$ and $\mu$. This is for instance the case for the usual de Rham or Dolbeault complexes.

\section{Acknowledgements}

I would like to thank Björn Jawerth, Alan McIntosh, Eugene Fabes and Steve Hofmann for several enriching conversations.

\section{References}

1. R. Brown, The method of layer potentials for the heat equation in Lipschitz cylinders, Amer. J. Math., 111 (1989), 339-379.

2. A. P. Calderón, The multipole expansion of radiation fields, J. Rat. Mech. Anal. 3 (1954), 523-537.

3. R. Coifman, A. McIntosh, and Y. Meyer, L'intégrale de Cauchy définit un opérateur borné sur $L^{2}$ pour les courbes Lipschitziennes, Ann. of Math. 116 (1982), 361-387.

4. D. Colton and R. Kress, Integral equation methods in scattering theory, Wiley, New York, 1983.

5. B. Dahlberg and C. Kenig, Hardy spaces and the $L^{p}-$ Neumann problem for Laplace's equation in a Lipschitz domain, Ann. of Math. 125 (1987), 437-465.

6. R. Dautray and J.-L. Lions, Mathematical Analysis and Numerical Methods for Science and Technology, vol. 1-4, Springer-Verlag, Berlin Heidelberg, 1990.

7. E. Fabes, M. Sand and J. Seo, The spectral radius of the classical layer potentials on convex domains, in: Partial differential equations with minimal smoothness and applications, 129-137, Springer, New York, 1992.

8. B. Jawerth and M. Mitrea, Higher dimensional scattering theory on $C^{1}$ and Lipschitz domains, preprint.

9. D. Jerison and C. Kenig, Boundary value problems on Lipschitz domains, in "Studies in Partial Differential Equations", Studies in Mathematics, MAA vol. 23, p.1-68, Washington D.C., 1982.

10. C. Kenig, Elliptic boundary value problems on Lipschitz domains, Beijing Lectures in Harmonic Analysis, Annals of Math. Studies 112 (1986), 131-183.

11. M. Mitrea, Clifford Wavelets, Singular Integrals, and Hardy Spaces, Lecture Notes in Mathematics, No. 1575, Springer-Verlag, 1994.

12. M. Mitrea, R. Torres and G. Welland, preprint.

13. C. Müller, Grundprobleme der Mathematischen Theorie elektromagnetischer Schwingungen, Springer-Verlag, Berlin, 1957. 
14. G. Verchota, Layer potentials and boundary value problems for Laplace's equation in Lipschitz domains, J. Funct. Anal. 59 (1984), 572-611.

15. H. Weyl, Kapazität von Strahlungsfeldern, Math. Zeit. 55 (1952), 187-198.

Institute of Mathematics of the Romanian Academy, P.O. Box 1-764, RO70700 BUCHAREST, ROMANIA

School of Mathematics, University of Minnesota, 127 Vincent Hall, 206 Church St. S.E., Minneapolis, MN 55455

E-mail address: mitrea@math.umn.edu 